\title{
Role of quantum vortices in atomic scattering from single adsorbates
}

\author{
A. S. Sanz ${ }^{*}$ and F. Borondo ${ }^{\dagger}$ \\ Departamento de Química, C-IX, Universidad Autónoma de Madrid, Cantoblanco, 28049 Madrid, Spain
}

S. Miret-Artés

Instituto de Matemáticas y Física Fundamental, Consejo Superior de Investigaciones Científicas, Serrano 123, 28006 Madrid, Spain

(Received 24 September 2003; revised manuscript received 25 November 2003; published 18 March 2004)

The scattering of $\mathrm{He}$ atoms from adsorbed $\mathrm{CO}$ molecules on the $\mathrm{Pt}(111)$ surface is described within the formalism of quantum trajectories provided by Bohmian mechanics. We show that the main mechanism leading to the formation of quantum rainbows and resonance enhanced trapping is quantum vortices.

DOI: 10.1103/PhysRevB.69.115413 PACS number(s): 79.20.Rf, 34.50.Dy, 34.10.+x, 03.65.Ta

\section{INTRODUCTION}

Adsorbates or other defects greatly affect the physics and chemistry of surfaces. They cause, for example, scattering intensity peaks in between Bragg angles, giving rise to diffusive scattering. ${ }^{1}$ This phenomenon is characterized by an incoherence among particles scattered from different defects, ${ }^{2}$ and is well illustrated by He scattering from $\mathrm{CO}$ molecules adsorbed on the $\mathrm{Pt}(111)$ surface, for which there is a wealth of experimental data and theoretical work. ${ }^{1,3-5} \mathrm{La}-$ hee et al. ${ }^{1}$ reported for the first time large-angle diffraction oscillations, explaining them in terms of the so-called reflection symmetry interference. This mechanism arises from the combination of adsorbate/surface double collisions, and direct scattering with the adsorbate. ${ }^{1,4}$ However, this interpretation, based on a hard-wall model, does not account for rainbow effects, which appear when realistic potentials are considered. ${ }^{3,5}$ Despite exhaustive work, some controversy regarding the assignment of the dynamical origin of diffraction peaks has been unavoidable. Arguments based on classical trajectories $^{3}$ are not conclusive by their own nature. On the other hand, those based on pure quantum calculations lack intuitive insight. ${ }^{5}$ Here we show by means of Bohmian mechanics ${ }^{6}$ that the dynamics of this process is strongly driven by transient quantum vortices.

Bohmian mechanics combines both the accuracy of the standard quantum description and an intuitive vision derived from a trajectory formalism, thus constituting a powerful tool to understand the physics underlying microscopic phenomena. In our case, a priori we may expect regular dynamics for trajectories colliding with the clean Pt surface. In a hydrodynamical view, this corresponds to a laminar regime. In contrast, the presence of the adsorbate will induce a more turbulent dynamics, leading to the formation of vortices that will play a key role. The theory of quantum vortices was first described by Dirac in a classic paper, ${ }^{7}$ and it has been shown that the quantization condition leading to their formation can be applied, in general, to many quantum phenomena, ranging from microscopic to macroscopic scales. In particular, here we show the important role that these vortices play in surface science, just as they do in other contexts, as superconductivity, Bose-Einstein condensation, or the Aharonov-Bohm effect.

The Bohmian formalism has been recently applied suc- cessfully to different problems. ${ }^{8,9}$ For example, Lopreore and Wyatt $^{10}$ have analyzed wave packets tunneling through barriers, nicely elucidating the corresponding mechanism in terms of quantum trajectories; a portion of them, in the foremost part of the initial packet, overcomes the barrier because they acquire some kinetic energy during a boost phase, shortly after the launching. With a similar analysis in mixed quantum-classical simulations, Prezhdo and Brooksby ${ }^{11}$ have solved the quantum back reaction problem for the reaction of $\mathrm{O}_{2}$ with $\mathrm{Pt}$, and Gindensperger et al. have reported a study including continuum states, ${ }^{12}$ and a description of rotational diffractive scattering. ${ }^{13}$

The organization of the paper is as follows. In Sec. II we briefly introduce the fundamentals of Bohmian mechanics and its connection with quantum vortices. In Sec. III the working model is described. In Sec. IV we present our quantum calculations, and their discussion. Finally, in Sec. V the main conclusions obtained from this work are summarized.

\section{QUANTUM VORTICES IN BOHMIAN MECHANICS}

Within the Bohmian formalism, ${ }^{6}$ the fundamental equations of motion are derived by introducing the wave function in polar form

$$
\Psi=R e^{i S / \hbar},
$$

into the time-dependent Schrödinger equation, thus obtaining

$$
\begin{gathered}
\frac{\partial R^{2}}{\partial t}+\nabla \cdot\left(R^{2} \frac{\nabla S}{m}\right)=0, \\
\frac{\partial S}{\partial t}+\frac{(\nabla S)^{2}}{2 m}+V-\frac{\hbar^{2}}{2 m} \frac{\nabla^{2} R}{R}=0,
\end{gathered}
$$

which are the continuity and quantum Hamilton-Jacobi equations, respectively; here $m$ is the incoming particle mass, $V$ the interaction potential, and the last term on the left-hand side of Eq. (2b) is the so-called quantum potential. This context-dependent nonlocal potential determines, together with $V$, the total force acting on the system.

In analogy to the classical Hamilton-Jacobi theory, quantum trajectories are defined by

$$
\mathbf{p}=\nabla S
$$


where $\mathbf{p}=m \dot{\mathbf{r}}$. The condition for these trajectories to form quantum vortices comes from the property of nonsingle valuedness of $S$, implied by the complex character of the wave function. Thus, discrete jumps in $S$ may only take place at nodal points, where the $\mathbf{p}$ field is rotational, since

$$
\oint d S=\oint \mathbf{p} \cdot d \mathbf{l}=\int(\boldsymbol{\nabla} \times \mathbf{p}) \cdot d \mathbf{A}=2 \pi \hbar n,
$$

with $n$ a nonzero integer. This condition gives rise to quantum streamlines that correspond to closed paths around nodes.

\section{THE POTENTIAL MODEL}

As stated above, the purpose of the present paper is to show the important role played by quantum vortices in the dynamics of the scattering process, as the mechanism responsible for the formation of the different features displayed by the intensity pattern. Unlike the classical and semiclassical mechanisms proposed in the literature, ${ }^{1,3-5}$ by means of Bohmian mechanics those features can be explained from a purely quantum perspective. Accordingly, we have chosen as a working model a linear defect ${ }^{1,3}$ instead of a point one. ${ }^{5}$ Due to the axial symmetry of the former, when incidence perpendicular to its axis is considered, calculations simplify since the model reduces to two dimensions, and (Bohmian) dynamical results are equivalent to those produced by a point defect (see below). Notice that this symmetry makes that the two-dimensional model associated to a transversal plane of the system leads to the same intensity pattern obtained from the full three-dimensional model. In this sense, to study the dynamics of quantum trajectories contained in a transversal plane is equivalent to do it considering the dynamics associated to the three-dimensional model. Unless the symmetry of the potential is broken by means of imperfections, incidence conditions (i.e., incidence out of the perpendicular direction), or thermal effects, for example, the quantum trajectories contained in a plane will remain in it, and will not mix with trajectories contained in other planes.

Nevertheless, it is worth mentioning that the dynamics associated to a point defect may be also studied by means of the same two-dimensional model (whenever the plane chosen is radial and perpendicular to the clean surface). In this case, quantum trajectories display the same features as those related to the two-dimensional model of the linear defect. However, unlike the case of the linear defect, the statistics of quantum trajectories to obtain the corresponding intensity pattern must be done by considering the full threedimensional model due to its symmetry. This is equivalent to take into account a large number of radial planes, since the trajectories belonging to a plane remain within the same plane, without mixing with trajectories contained in other radial planes (this is similar to what happens in the case of the linear defect, as seen above). Thus, these trajectories may diffuse along two directions, while in the two-dimensional model of the linear defect diffusion takes place only along one direction. Hence, noticeable differences are observed in the peaks of the intensity patterns corresponding to these models. $^{5}$

Taking into account the previous statements, the classical model that we have used in our calculations consists of a two-dimensional Hamiltonian

$$
H=\frac{1}{2 m_{\mathrm{He}}}\left(p_{x}^{2}+p_{z}^{2}\right)+V(x, z),
$$

where $x$ and $z$ are, respectively, coordinates along the parallel and perpendicular directions to the clean Pt surface, and measured relative to the $\mathrm{CO}$ center of mass. The twodimensional potential model is that used by Yinnon et al. ${ }^{3}$ for this type of scattering process, and that has been also recently used to perform quantum dynamical calculations. ${ }^{5}$ It consists of the sum of a Morse function for the He-Pt(111) process,

$$
V_{\mathrm{He}-\mathrm{Pt}}(z)=D_{s}\left[e^{-2 \alpha\left(z-z_{m}\right)}-2 e^{-\alpha\left(z-z_{m}\right)}\right],
$$

with $D_{s}=4.0 \mathrm{meV}, \alpha=0.6 \mathrm{bohr}^{-1}$, and $z_{m}=2.3 \mathrm{bohr}$, and a Lennard-Jones function for the He-CO interaction,

$$
V_{\mathrm{He}-\mathrm{CO}}\left(\mathbf{r}-\mathbf{r}_{\mathrm{CO}}\right)=4 \epsilon\left[\left(\frac{\sigma}{r}\right)^{12}-\left(\frac{\sigma}{r}\right)^{6}\right],
$$

where $\epsilon=2.37 \mathrm{meV}$ and $r_{0}=3.5 \AA\left(r_{0}=2^{1 / 6} \sigma\right)$. Notice that, due to the slight corrugation of the $\mathrm{Pt}(111)$ surface, ${ }^{3}$ the He$\mathrm{Pt}(111)$ interaction is described by a "flat" potential along the $x$ direction.

\section{RESULTS AND DISCUSSION}

In order to simulate with good accuracy the scattering process, we use an initial incoming plane wave, approximated as a linear superposition of 250 Gaussian wave packets, ${ }^{9}$ homogeneously distributed along a distance of 100 bohr. The width parameters of each wave packet are taken as $\sigma_{x}=1.58 \mathrm{bohr}$ and $\sigma_{z}=5 \mathrm{bohr}$, respectively. The resulting plane wave is then launched from $\langle z\rangle_{0}=19.4 \mathrm{bohr}$ (where the classical potential is negligible) with a normal momentum $k_{i}=2.32 \mathrm{bohr}^{-1}$. This corresponds to an initial energy $E_{i}=10 \mathrm{meV}$, and a de Broglie wavelength $\lambda_{\mathrm{dB}}=2.71 \mathrm{bohr}$. The time-dependent Schrödinger equation is then solved by a standard spectral method, ${ }^{14}$ and quantum trajectories are obtained by numerical integration of Eq. (3).

The results of our calculations are shown in Fig. 1, where panel (a) displays the diffraction intensity, computed by standard $S$-matrix methods. ${ }^{15}$ Two sets of results are shown. The dash line corresponds to the full calculation, while the solid one gives the results after the contribution of the scattering due to the clean Pt surface has been removed. ${ }^{15}$ This procedure eliminates oscillations at small deflection angles $\theta_{\text {def }}$, which disappear by destructive interference in a periodic surface. ${ }^{16}$ The different peaks have been labeled as $A_{1,2,3}, B$, and $C+D$, indicating their different origins. A snapshot of the probability density is shown in Fig. 1(b), where a series of maxima, correlated with peaks in (a), are seen. The existence of many maxima in this region at small values of $\theta_{\mathrm{def}}$ (peaks $A$ ) is due to the contribution of the clean Pt surface, as indicated above. The three remaining lobes correspond to 

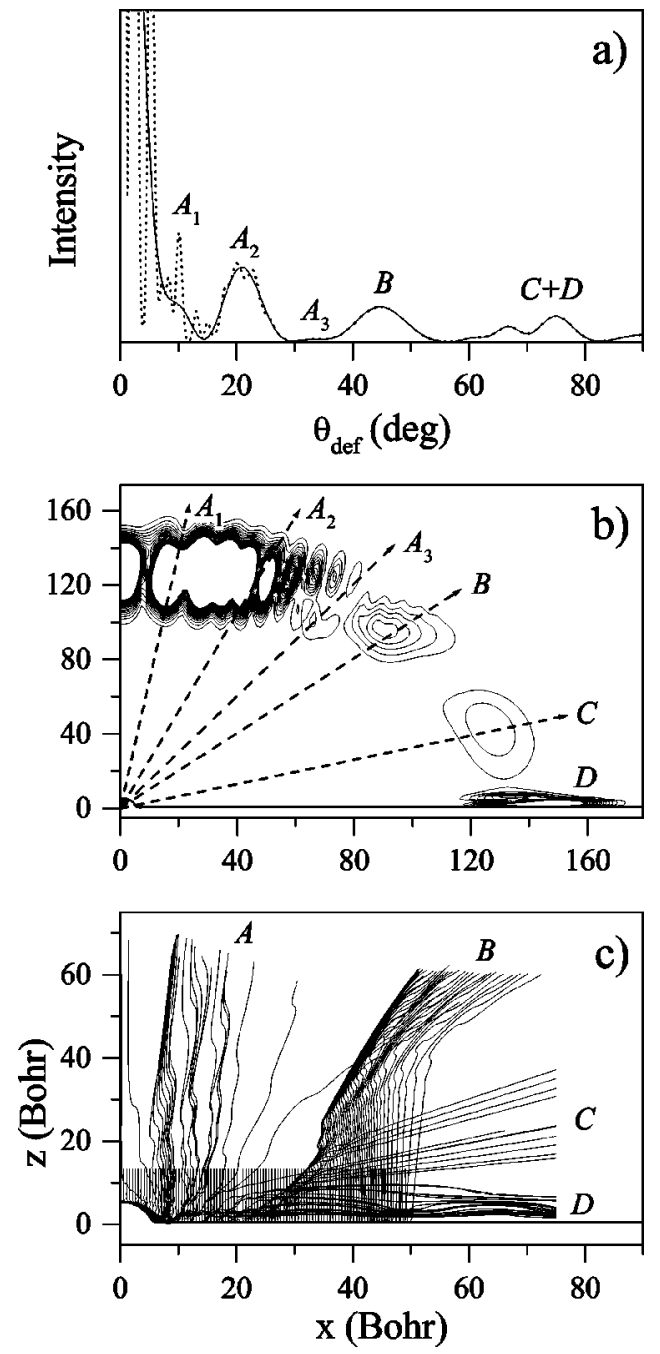

FIG. 1. (a) Relative intensity as a function of the deflection angle for $\mathrm{He}-\mathrm{CO} / \mathrm{Pt}(111)$ scattering at $E_{i}=10 \mathrm{meV}$ and normal incidence. (b) Probability density at $t=11 \mathrm{ps}$. The different maxima, whose positions are indicated by dash arrows, correlate with peaks in the upper panel. (c) Quantum trajectories whose initial positions are distributed parallel to the surface at $z_{0}=\langle z\rangle_{0}-6$ bohr.

peaks $B$ and $C$, assigned to rainbow features in the literature, ${ }^{3,5}$ and to the portion of the wave function trapped on the surface, labeled as $D$. This surface trapping in the bound channel of the Morse function takes place through a very effective $z-x$ kinetic-energy transfer due to interaction with the boss, constituted by the adsorbed $\mathrm{CO}$ molecule, and has been called rainbow-enhanced trapping by Yinnon et al. ${ }^{3}$ The bottom part of Fig. 1 shows the corresponding quantum trajectories. To make the plot less congested we have only included trajectories corresponding to a cut in the initial wave function, parallel to the surface, taking their initial positions $\left(x_{0}\right)$ homogeneously distributed along it. At first sight, this figure shows a very complex dynamics. However, it is easily appreciated in their asymptotic parts that quantum trajectories appear grouped in clumps around the deflection angles corresponding to the different peaks in Fig. 1(a).

To analyze the results of Fig. 1(c) in more detail, in Fig. 2 we show a blownup of these trajectories separated in differ-
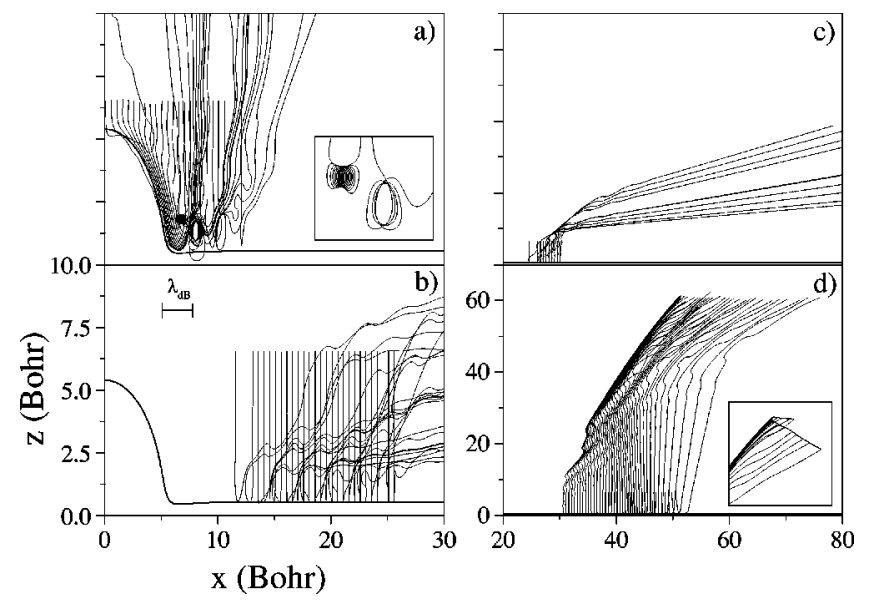

FIG. 2. Blownup of quantum trajectories shown in Fig. 1(c).

ent groups. As $x_{0}$ increases, different groups of trajectories can be identified. The dynamics of trajectories in part (a), starting close to the origin, is dictated by the presence of the $\mathrm{CO}$; trajectories initially slide along the boss, performing later a complicated motion close to the surface, and leave the interaction region at small values of $\theta_{\text {def }}$. In the Fraunhofer region ${ }^{16}$ these trajectories contribute to peaks $A$. In Fig. 2(b) all trajectories become trapped after interacting with the surface, constituting group $D$, and showing a relatively complex dynamics. This effect, solely due to the quantum potential, is however quite surprising from the point of view of classical treatments, since taking place at distances where the $\mathrm{He}-\mathrm{CO}$ interaction is negligible. For bigger values of $x_{0}$ [Figs. 2(c) and 2(d)] dynamics simplify, corresponding to a direct scattering in which trajectories are just deflected from the surface. As this happens, they leave the surface in two groups at angles corresponding to peaks $B$ [part (c)] and $C$ [part (d)]. It is interesting to note that the deflection angle for the latter closely resembles the behavior of a classical rainbow because of the existence of a caustic, clearly appreciated in the inset. Two points are worth discussing here. First, as indicated above, the behavior of trajectories shown in Figs. 2(a) and 2(b) is much more complex than that in (c) and (d). Actually, it can be said that the latter corresponds to a laminar regime, while the former is turbulent, with a smooth transition between them in close analogy with classical fluid dynamics. ${ }^{8,9}$ Second, and more importantly, we see in (a) and (b) that the complex dynamics mentioned above is not completely chaotic, but gets highly organized around a number of points avoided by the trajectories. These points constitute centers around which trajectories loop [see inset to Fig. 2(a)] showing transient trapping, and have been identified as quantum vortices. Notice that these quantum vortices appear evenly spaced at intervals given approximately by $\lambda_{\mathrm{dB}}$, and that they are located along straight lines parallel to the $\mathrm{Pt}$ surface, two of which can be distinguished in the figure. Let us remark that this transient vortitial trapping should not be confused with trapping associated to group $D$.

Figure 3 shows how these nodes form, by presenting three snapshots of the wave function as it evolves at the adsorbate vicinity. We see that, as it gets diffracted by the boss [part (a)], a series of spherical outgoing wave fronts forms. Sub- 


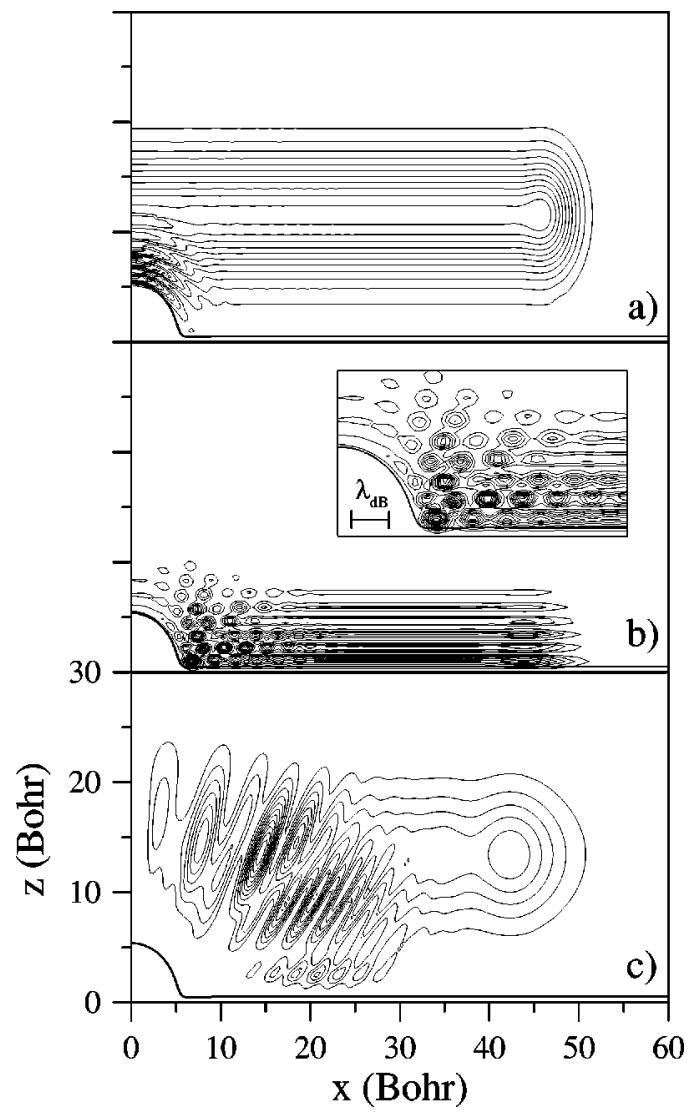

FIG. 3. Snapshots of the wave function in the vicinity of the interaction region, showing the formation of a nodal pattern which gives rise to the quantized vortices influencing the quantum trajectories of Fig. 1(c).

sequently, when the wave reaches the clean Pt surface another series of flat, parallel, superposing wave fronts appear [part (b)]. This interference process originates a pattern of nodal lines, separated by approximately $\lambda_{\mathrm{dB}}$, which is clearly appreciated in the inset, and vortices take place at the intersections. Later, this pattern dilutes as the outgoing wave evolves towards the asymptotic region [part (c)].

In contrast with a classical approach, the dependence of results on the particle initial conditions is an important issue in our problem, due to the nonlocal character of Bohmian mechanics. Accordingly, we consider in Fig. 4 trajectories started at a different cut than the one used in Fig. 1(c). Some important differences are worth commenting. First, these trajectories do not get so close to the interaction region due to the existence of an effective barrier, that can be alternatively interpreted as a quantum pressure caused by other trajectories in the ensemble. ${ }^{9}$ This implies a weaker interaction acting on the incoming particles, and thus simpler dynamics. As a result, the regime is not so turbulent, and a wide region of laminar flow is appreciated. The influence of the vortitial dynamics is nevertheless felt. Second, these trajectories contribute mainly to diffraction peaks $A$, leaving the scattering scenario through the "hole" in $\theta_{\text {def }}$ left by trajectories of Fig. 1(c).

An additional important result appears when the overall outgoing structure of Fig. 4 is considered, where a moire

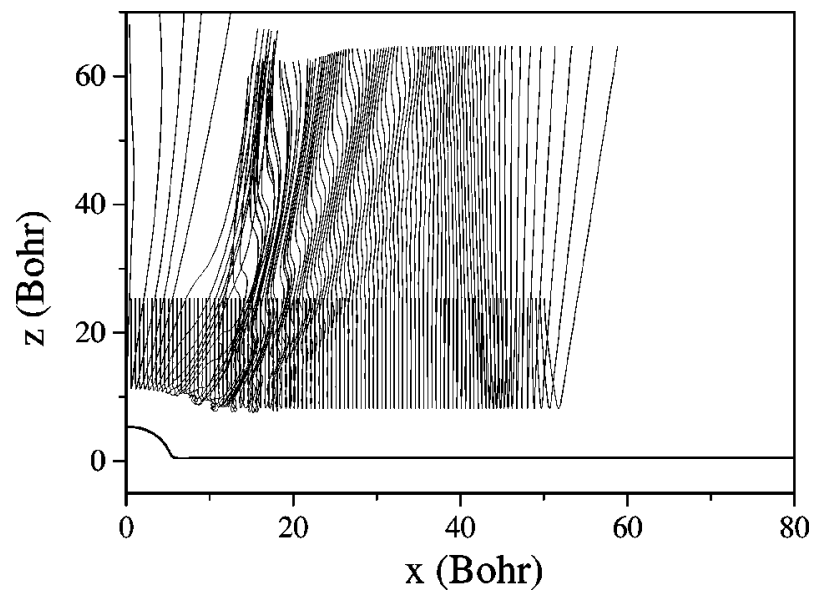

FIG. 4. Quantum trajectories whose initial positions are distributed parallel to the surface at $z_{0}=\langle z\rangle_{0}+6$ bohr.

pattern, consisting of a series of dark fringes, is observed. As explained in Ref. 16 these accumulations of trajectories are induced by large variations in the quantum potential, making trajectories pushing each other when they try to cross. What is relevant to our work is the fact that, when considering the whole ensemble of trajectories representative of the initial wave, this effect also occurs with trajectories started in other cuts, such as those in Figs. 2(c) and 2(d). As a result, there is a causal connection between the region of the potential close to the boss and the rainbow exit region.

Finally, to further stress the importance of vorticity, we have estimated from the binning ${ }^{16}$ the percentage of vortitial trajectories contributing to each diffraction peak. The results are $29.9 \%$ for peaks $A, 20.9 \%$ for $B$, and $100 \%$ for both $C$ and $D$, which represents in all cases a substantial fraction of the intensities. Notice, moreover, that these trajectories undergo delays in time-of-flight measurements. Due to their trapping around quantum vortices, trajectories directly scattered will reach the detector before than those temporary trapped. On the other hand, additional effects may appear in the case of charged incoming particles (low-energy electrons, for example), as transient magnetic currents generated by particles looping over the surface (close to the adsorbate).

\section{CONCLUSIONS}

To summarize, in this work we give a description of atomic scattering from single adsorbates using Bohmian mechanics, which provides an intuitive causal vision of this phenomenon within a fully quantum framework. We have chosen a system for which classical rainbow conditions are given. Here, rainbow features are associated with the whole diffraction pattern rather than with individual peaks, ${ }^{17}$ which can be understood from the nonlocal character of the quantum potential, causing the adsorbate to extend its influence further than its classical range of interaction. The process takes place through a very interesting quantum dynamics, in which the concept of transient vortitial trapping plays a key role. In this way, we provide a more complete description of the corresponding scattering process than exists in the literature. Bohmian trajectories show how quantum results go be- 
yond the customary classical notions, helping to understand the quantum dynamics leading to the different diffraction peaks, thus avoiding the controversy mentioned in the Introduction. Within our description all features displayed by the intensity pattern can be well explained by means of elements contained in the formal structure of Bohmian mechanics, without any need to consider another external concepts, as it happens with the classical and semiclassical mechanisms proposed in the literature.

\section{ACKNOWLEDGMENTS}

This work was supported in part by MCyT (Spain) under Contracts Nos. BFM2000-347, BFM2001-2179, BQU2001147, and BQU2003-8212. Authors thank Professor Turgay Uzer for useful suggestions and a critical reading of the manuscript. A.S.S. gratefully acknowledges the Consejería de Educación y Cultura of the Comunidad Autónoma de Madrid (Spain), for financial support.
*Email address: angel.sanz@uam.es

†Corresponding author. Email address: f.borondo@uam.es

†Email address: salvador@fam10.imaff.csic.es

${ }^{1}$ A.M. Lahee, J.R. Manson, J.P. Toennies, and Ch. Wöll, Phys. Rev. Lett. 57, 471 (1986); J. Chem. Phys. 86, 7194 (1987).

${ }^{2}$ B. Poelsema and G. Comsa, Scattering of Thermal Energy Atoms from Disordered Surfaces (Springer, Berlin, 1989).

${ }^{3}$ A.T. Yinnon, R. Kosloff, and R.B. Gerber, J. Chem. Phys. 88, 7209 (1988).

${ }^{4}$ B.H. Choi, K.T. Tang, and J.P. Toennies, J. Chem. Phys. 107, 9437 (1997).

${ }^{5}$ M.N. Carré and D. Lemoine, J. Chem. Phys. 101, 5305 (1994); D. Lemoine, Phys. Rev. Lett. 81, 461 (1998).

${ }^{6}$ P.R. Holland, The Quantum Theory of Motion (Cambridge University Press, Cambridge, 1993).

${ }^{7}$ P.A.M. Dirac, Proc. R. Soc. London, Ser. A 133, 60 (1931).

${ }^{8}$ F. Sales Mayor, A. Askar, and H.A. Rabitz, J. Chem. Phys. 111,
2423 (1999).

${ }^{9}$ A.S. Sanz, F. Borondo, and S. Miret-Artés, J. Phys. C 14, 6109 (2002).

${ }^{10}$ C.L. Lopreore and R.E. Wyatt, Phys. Rev. Lett. 82, 5190 (1999).

${ }^{11}$ O.V. Prezhdo and C. Brooksby, Phys. Rev. Lett. 86, 3215 (2001).

${ }^{12}$ E. Gindensperger, C. Meier, and J.A. Beswick, J. Chem. Phys. 116, 8 (2002).

${ }^{13}$ E. Gindensperger, C. Meier, J.A. Beswick, and M.-C. Heitz, J. Chem. Phys. 116, 10051 (2002).

${ }^{14} \mathrm{R}$. Kosloff, in Dynamics of Molecules and Chemical Reactions, edited by R.E. Wyatt and J.Z. Zhang (Marcel Dekker, New York, 1996), pp. 185-230.

${ }^{15}$ G. Drolshagen and R. Vollmer, J. Chem. Phys. 87, 4948 (1987).

${ }^{16}$ A.S. Sanz, F. Borondo, and S. Miret-Artés, Phys. Rev. B 61, 7743 (2000).

${ }^{17}$ A.S. Sanz, F. Borondo, and S. Miret-Artés, Europhys. Lett. 55, 303 (2001). 